\title{
Perfil competencial requerido en el mercado español del gran consumo a los graduados en marketing: un análisis de contenido de las ofertas de empleo en Internet
}

\author{
Jorge García Martín" y Lázaro Echegaray \\ CÁMARABILBAO University Business School
}

\begin{abstract}
Resumen
Objetivo: El objetivo del estudio es definir las competencias que actualmente aparecen en las ofertas de empleo del mercado de gran consumo, destinadas a graduados en marketing, como respuesta de la empresa a los cambios que la globalización y el desarrollo tecnológico vienen produciendo en los mercados. Metodología: Esta investigación se ha abordado desde una metodología mixta. La primera fase se ha basado en el rastreo de fuentes documentales con técnica Desk Research en los dos portales de empleo con más notoriedad en España: Infoempleo e Infojobs. El siguiente paso ha sido el análisis de contenido y la cuantificación de cada uno de los requisitos aparecidos en las ofertas de empleo analizadas. Resultados: Los resultados hablan de la importancia del uso de las Redes Sociales en lo referente a las competencias digitales, de la relevancia del idioma inglés dentro de las metahabilidades, y del dominio de los planes de marketing en relación a los requisitos propios de los fundamentos del marketing. En lo que tiene que ver con la concordancia experiencia-salario, se ha observado una alta relación entre estas dos variables. Limitaciones: En tanto que la investigación se ha centrado en el mercado B2C, las conclusiones no son aplicables directamente a otros mercados. Implicaciones prácticas: El valor de la investigación reside en la importancia que para la formación universitaria tiene conocer las últimas competencias exigidas en el mercado, al objeto de adaptar sus planes de estudio.
\end{abstract}

Palabras clave: marketing; formación universitaria; perfil competencial; reclutamiento; competencias y habilidades.

Códigos JEL: M53.

\footnotetext{
ISSN 0212-1867 / e-ISSN 1989-3574

(C) ESIC Editorial, ESIC Business \& Marketing School

DOI: $10.7200 /$ esicm.162.0501.3e

http://www.esic.edu/esicmarket
}

"Autor de correspondencia. e-mail: jorge.garcia@camarabilbaoubs.com 


\section{Introducción}

La rotación de empleados es un fenómeno ampliamente estudiado en la gestión de recursos humanos y otras disciplinas relacionadas con la gestión de personal en organizaciones (Buttner \& Lowe, 2017; Johnson \& Yanson, 2018; Salin \& Notelaers, 2017; DiPietro \& Bufquin, 2018). La rotación a menudo es una gran pérdida para las organizaciones, tanto en términos de capital humano como en el coste que se deriva de la salida de un empleado y el proceso de contratación de un nuevo empleado (Heavey, Holwerda, \& Hausknecht, 2013). La literatura existente ha reconocido que las altas tasas de rotación afectan negativamente a las operaciones diarias de la organización y al rendimiento general, y en consecuencia amenazan su éxito y sostenibilidad (Alola, Avci, \& Ozturen, 2018; Bok, 1995; Brereton, Beach \& Cliff, 2003; Brymer \& Sirmon, 2018; Leana III, y Van Buren, 1999). Dada la importancia de la rotación de los empleados para determinar el éxito o el fracaso de una organización, el tema ha recibido una atención sustancial de los gerentes de negocios e investigadores (Ton \& Huckman, 2008).

Los entornos complejos y dinámicos en los que se mueven hoy en día las empresas generan un alto grado de incertidumbre con arreglo a conocer los escenarios donde deben competir, no solo en el medio plazo, sino incluso en el corto. Así, el marketing vive inmerso en la preocupación por interpretar convenientemente dicho entorno para adaptarse a los cambios de la manera más ventajosa posible, algo que modifica continuamente las habilidades y competencias requeridas a los profesionales. A juicio de Ventura (2013), la globalización de la economía, la cual conlleva una incesante internacionalización de las actividades de la empresa, junto con las nuevas tecnologías, generan hoy en día entornos turbulentos derivados de esa complejidad y dinamismo.

Ante este marco situacional cabe plantearse cómo afectan estos condicionantes a las políticas de marketing de las empresas, y más concretamente a las competencias y habilidades que actualmente demandan estas para cubrir los diferentes puestos ofertados. Precisamente, esta será la principal preocupación de la presente investigación, si bien concretándola en los requerimientos demandados exclusivamente en el mercado del gran consumo. Se pretende, por tanto, obtener una información sobre las competencias requeridas que no esté "contaminada" por las demandas del mercado organizacional. En este sentido, estudios como el de García y Echegaray (2018) han ofrecido un perfil sobre los requerimientos del profesional del marketing en el mercado nacional desde una perspectiva general, dejando la puerta abierta para investigar las diferencias que pueda haber entre el mercado del gran consumo y el organizacional.

Son diversos los autores que han estudiado las competencias y habilidades requeridas por los departamentos de marketing y su adecuación o no a los planes de formación de las escuelas de negocios, universidades y centros universitarios (en adelante formación universitaria), y lo han hecho además desde diferentes ópticas y aproximaciones de las que este artículo se ocupará más adelante. 
Sin embargo, tras analizar la literatura al respecto, se echa en falta una investigación concreta o una aproximación más específica dirigida al conocimiento de las destrezas requeridas en el mercado de consumo (en adelante B2C). Consecuentemente, la presente investigación dirigirá su atención al análisis de las competencias y habilidades demandadas a los profesionales del marketing en dicho mercado, contribuyendo con sus aportaciones, y en la medida de sus recursos, al mejor conocimiento de este campo. Asimismo, la investigación tratará de establecer la relación respecto al grado de experiencia requerido y la remuneración ofrecida a los candidatos que optan por trabajar en dicho mercado B2C.

Para alcanzar estas metas, la investigación recurrirá a las ofertas publicadas por los portales de empleo más relevantes en el mercado español, un campo este poco investigado aún, en un momento en el que tanto el volumen de procesos de selección para profesionales del marketing como la cantidad de oferta de empleo disponible han aumentado con respecto al 2016 (Dottle, 2018; Michael Page, 2018).

Se espera que los descubrimientos detectados en la investigación aporten valor a la formación universitaria, la cual contará con una información relevante para orientar sus planes de estudio especializando su oferta formativa hacia el B2C. Asimismo, será posible también mejorar las competencias de aquellos alumnos que opten por orientar su carrera profesional hacia dicho mercado.

De igual forma se espera también que el estudio contribuya a aumentar la cooperación, necesaria aunque insuficiente hasta ahora (Royle y Laing, 2014), entre el mundo académico y la empresa por lo que al marketing respecta.

\section{Marco teórico}

El impacto originado tanto en el macro como en el microentorno por la globalización y la revolución tecnológica está provocando en el marketing cambios significativos en el conocimiento y en las habilidades empleadas para poder enfrentarse con garantías a una realidad más competitiva y desafiante (Schibrowsky, Peltier y Boyt, 2002).

De acuerdo con Santesmases (2007), debido a la utilización generalizada de Internet, de las nuevas tecnologías y de las bases de datos relacionadas, el viejo marketing de transacciones se ha convertido en un marketing de relaciones. Sin embargo, el progreso de las habilidades personales en el entorno del marketing digital se encuentra con ciertas dificultades en no pocas organizaciones. Los problemas derivados de la cultura corporativa y de las habilidades organizacionales, a juicio de Bakhshi y Mateos-García (2012), suponen un nuevo reto para el desarrollo de las destrezas en el marketing digital, situación que se ve a su vez agravada por el dinamismo y la cantidad de herramientas analíticas y de medición existentes en el mercado.

Antes de conocer cuáles son las habilidades y competencias necesarias en el marketing recogidas por la literatura, consecuencia de este nuevo escenario, es necesario concretar el significado que la presente investigación ofrece del término "competencia" en consonancia con la literatura encontrada. 
Así, tal y como señala Palomo (2008), la relativa corta historia del concepto ha deparado por parte de los autores que se han preocupado por el mismo una divergencia respecto a su significado. A la vista de la diversidad de definiciones encontradas desde el año 1982 recogidas por Palomo (2008), se aprecian en el término "competencia" tres elementos principales inherentes al mismo: las conductas, los conocimientos y las habilidades. Dicho esto, son muchos los autores que utilizan indistintamente estos tres elementos (Leeflang et al., 2014; Royle y Laing, 2014; Pefanis y Harich, 2010; Fuentes, 2009), habiendo incluso otros que añaden nuevos como por ejemplo el de "atributos" o el de "requerimientos" para referirse a las competencias demandadas por las organizaciones a sus profesionales.

No es infrecuente comprobar cómo muchos de los estudiantes que concluyen su grado universitario en marketing ven que son pocos los trabajos en los que pueden usar del conocimiento y de las técnicas aprendidas en la facultad (Pefanis y Harich, 2010). A estas conclusiones se suman las extraídas de la investigación de Harraway y Barker (2005), para quienes los estudiantes con grados en marketing, economía y finanzas, manifiestan importantes divergencias entre lo requerido en el trabajo y lo aprendido en la universidad.

Ante esta situación, los autores son partidarios de unir el conocimiento conceptual a las habilidades prácticas con objeto de alcanzar el compromiso educativo óptimo para el alumno. En este mismo contexto, otros estudios como el de Davis, Misra y Van Auken (2002) aseguran que los alumnos de marketing se ven asimismo poco preparados en habilidades y sobrepreparados en áreas de conocimiento designadas.

Revisada la literatura, se ha comprobado cómo una parte significativa de los investigadores (Ortiz, Joyanes y Giraldo, 2016; Royle y Laing, 2014; Leeflang et al., 2014; Day, 2011; Brady, Fellenz y Brookes, 2008) dirige su análisis al área digital y a las tecnologías de la información, mientras otra lo hace hacia la pequeña y mediana empresa (Boam y Sparrow, 1992; Gilmore, Carson y Grant, 2001). También se han encontrado otras aproximaciones al análisis de las competencias y habilidades más dirigidas hacia los puestos y responsabilidades concretas como la de product manager o brand manager, entre otras (Wroblowská, 2016; Wroblowská y Ruda, 2015; Foxize School, 2014; Pefanis y Harich, 2010; Palomo, 2008), o enfoques orientados a la investigación de marketing y al desarrollo de nuevos productos (McNally, Durmusoglu y Calantone, 2013; Micu et al., 2011; Carbonell-Foulquié, ManueraAlemán y Rodríguez-Escudero, 2004; Tzokas, Hultink y Hart, 2004).

En el campo de las tecnologías de la información y del marketing digital, estudios como el de Royle y Laing (2014) han delimitado siete habilidades o competencias clave, a saber: fundamentos de la comunicación corporativa, habilidades para mantener una buena relación con el cliente, capacidades para la previsión, así como para la elaboración de contingencias, con objeto de poder actuar en tiempo manejando herramientas como blogs, redes sociales o foros, competencias para el estudio del macroentorno, integración estratégica de las habilidades referidas al marketing digital (monitorización de datos, métricas...), y para finalizar un conocimiento tecnológico general (desarrollo web, SEO, edición de vídeo, aplicaciones móviles...). 
El uso de las herramientas y tecnologías digitales, el dominio de las nuevas métricas para la medición de resultados, la gestión de la marca en el entorno digital, o las habilidades analíticas, constituye otro grupo importante de capacidades detectadas por Leeflang et al. (2014) dentro de los requerimientos dirigidos a los profesionales del marketing en general, y producidos por los profundos y constantes cambios en el mercado. De igual forma, otras investigaciones como la de Brady, Fellenz y Brookes (2008) han puesto su atención en las tecnologías de la información y la comunicación, detectando un grupo de habilidades necesarias para los profesionales del marketing provenientes de la automatización de la fuerza de ventas. Concretamente, el empleo de herramientas como el EDI, sistemas de CRM, bases de datos, el Sistema de Información de Marketing o el entorno de Internet, y más específicamente el e-marketing, constituye el grupo de competencias citado por estos autores.

Otra categoría en la que la literatura ha centrado su interés es la constituida por la dimensión de la empresa, centrando su atención sobre las habilidades y competencias requeridas por las pequeñas y medianas organizaciones en relación a las grandes. Así, desde la perspectiva de Carson (1990), aquellas presentan una idiosincrasia distinta, lo cual hace que sus políticas de marketing no sean iguales, por lo que las habilidades y competencias interesadas no tienen por qué ser las mismas que para las grandes empresas.

En el ámbito de la pyme, el networking es presentado como una herramienta de suma importancia (Gilmore, Carson y Grant, 2001), cuyo desarrollo conlleva una mejora en las comunicaciones de la dirección de la compañía con sus competidores, incrementando la participación y el concurso, algo con lo que la empresa ve mejorada su posición competitiva. Otros estudios un poco más teóricos, como los de Carson y Gilmore (2000), han centrado las competencias en cuatro áreas: el conocimiento -fundamentalmente sobre el microentorno- la comunicación, la experiencia y el buen juicio, entendiendo este como la habilidad para medir la información obtenida de las tres habilidades citadas anteriormente y tomar así las decisiones más oportunas. Este grupo de competencias, de acuerdo con Carson y Gilmore (2000), se vería complementado con el aprendizaje experiencial.

Por lo que respecta a los estudios recogidos sobre el desempeño de las responsabilidades derivadas del área de marketing, tales como brand manager o product manager entre otras, en el elaborado por Foxize School (2014) a partir de 38 entrevistas a profesionales relevantes del sector en el mercado español se observa en los requerimientos un claro denominador común con seis aspectos, a saber: la creatividad, el liderazgo, las habilidades digitales, la flexibilidad, la empatía y la capacidad para trabajar en organizaciones líquidas, entendiendo este elemento como una competencia necesaria para la adaptación al cambio, y para estimular y acelerar el movimiento de la organización. Asimismo, en el estudio elaborado por García y Echegaray (2018) a partir de una muestra formada tanto por empresas internacionalizadas como por informantes de doble condición: docentes universitarios y directivos de marketing, se concluye en la necesidad de reorientar los planes de estudio de marketing en la formación universitaria española, para cubrir competencias demandadas por la 
empresa tales como las habilidades sociales y de comunicación, las destrezas para trabajar en organizaciones líquidas y las competencias propias del marketing digital. Siendo esto así, otras investigaciones en este mismo campo, como la realizada por Palomo (2008), han detectado un conjunto de siete competencias inherentes al director de marketing: la visión estratégica, la toma de decisiones, la integridad y honestidad, la orientación al cliente, la flexibilidad, la dirección y motivación de equipos y las relaciones con los demás. Igualmente, las investigaciones desarrolladas por Pefanis y Harich (2010) a partir de una muestra de 500 anuncios tomados de Monster.com, y centradas en el mercado estadounidense, han ordenado su análisis en base a tres grandes categorías: "habilidades técnicas", "metahabilidades" y "conocimiento de marketing". Así, dentro de cada una de ellas han recogido todos aquellos requerimientos especificados por las empresas en sus anuncios de empleo. En la primera categoría ("habilidades técnicas"), las más demandadas fueron las competencias en MS Office y Outlook; en la segunda ("metahabilidades"), la comunicación oral y escrita, y en la tercera, correspondiente al "conocimiento de marketing", la comprensión del comportamiento del consumidor, la publicidad y la gestión de las ventas. De otro lado, la investigación llevada a cabo en el mercado checoslovaco por Wroblowská y Ruda (2015) a partir de la recolección de anuncios sobre ofertas de empleo del portal Jobs.cz entre los meses de marzo y agosto de 2014, aporta su propia relación respecto de aquellas habilidades más habitualmente requeridas desde la empresa; a saber: las habilidades comunicativas, las propias de la negociación, las inherentes al trabajo en equipo, y las relacionadas con el pensamiento analítico.

Poco más tarde Wroblowská (2016), en una nueva investigación a partir de los datos obtenidos de una muestra de 56 anuncios de empleo del portal antes mencionado (Jobs.cz.), estableció cuatro grandes categorías para el análisis: "requerimientos educativos", "conocimiento técnico y habilidades", "competencias generales transferibles" y "práctica y experiencia". De la exploración estadística de las competencias aportadas para cada categoría, la investigadora concluyó en que las habilidades más demandadas para cada una de ellas respectivamente fueron las siguientes: el grado universitario, el conocimiento en disciplinas técnicas, el inglés como idioma y finalmente el grado de experiencia en marketing o en puestos similares al ofertado.

En el marco que comprende tanto a la investigación como al desarrollo de nuevos productos, Micu et al. (2011) ponen el acento en las transformaciones sufridas por las habilidades de investigación en marketing, consecuencia de la irrupción de la digitalización. Como resultado de todo ello se observa la aparición de nuevas habilidades en el ámbito laboral, diferentes a las tratadas por las investigaciones realizadas hasta el momento en el entorno del marketing general, el marketing digital y la pequeña y mediana empresa. Siendo esto así, McNally, Durmusoglu y Calantone (2013), centran su estudio en los rasgos de la personalidad, al entender que son un elemento determinante para gestionar la innovación digital, algo que según los propios investigadores acaba condicionando la toma de decisiones en la empresa.

Finalmente, respecto a la remuneración de los profesionales del marketing, la literatura viene centrando su atención en la fuerza de ventas, por ser uno de los 
principales problemas en las organizaciones comerciales (Torres, Tiffer y Sandoval, 2017; Cicera i Soler, 2009; Nomparte, 2003; Chiesa y Renart, 2000). No obstante, otros enfoques han centrado su atención sobre la remuneración del director de marketing (Bronwyn, 2003), si bien en mercados muy alejados del español. Llegados a este punto, las consultoras de recursos humanos se han mostrado como una fuente de información relevante para conocer los niveles salariales y las perspectivas respecto a los honorarios de los profesionales del marketing en el mercado nacional (Dottle, 2018; Michael Page, 2018; Randstad, 2018). El interés de estos informes es mayor por cuanto dividen la función de marketing en varios puestos o categorías (Dottle, 2018; Randstad, 2018), escogiendo cinco mercados como son Barcelona, Madrid, Bilbao, Sevilla y Valencia, ofreciendo la remuneración media para cada uno de ellos. Si bien dichos informes no ofrecen datos cuantitativos respecto a la correlación entre remuneración y experiencia, sí aportan una información relevante para comprender la evolución de los honorarios de los profesionales del marketing en el corto plazo y el papel que juega en ellos la experiencia. Así, dentro de los perfiles más solicitados, la experiencia profesional, sobre todo la centrada en el sector de referencia para la empresa, es un factor de máxima importancia en los requerimientos de la oferta con consecuencias en la remuneración (Dottle, 2018; Michael Page, 2018).

Como conclusión, es justo señalar que en la literatura estudiada se ha encontrado una extensa gama de capacidades y de habilidades solicitadas hoy por hoy desde las organizaciones en el ámbito del marketing, pudiéndose incluso establecer diferencias entre ellas dependiendo del enfoque de la investigación, bien desde un ámbito general, desde la pequeña y mediana empresa, desde lo digital o desde el ámbito de la investigación y el desarrollo de nuevos productos. Sin embargo, no ha sido posible encontrar ningún estudio que dirija su atención hacia la relación entre las habilidades requeridas y el tipo de mercado donde se realiza la transacción, bien sea este entre empresas o entre empresas y consumidores fundamentalmente. Esta circunstancia hace que el presente estudio cobre interés al preocuparse específicamente por las competencias y las remuneraciones asociadas a los años de experiencia, solicitadas en el mercado B2C.

\section{Objetivos de la investigación}

Esta investigación se ha llevado a cabo a partir del establecimiento de un objetivo general y unos objetivos específicos que se definen de la siguiente manera:

- Como objetivo general, la investigación plantea definir los requerimientos y las competencias que se exigen en las ofertas de empleo para graduados universitarios en marketing, publicadas en los principales portales de Internet dedicados a la búsqueda de empleo (Infojobs e Infoempleo), en el mercado B2C. El análisis de tales requerimientos se llevará a cabo en las cinco comunidades autónomas españolas con mayor aportación al PIB nacional en 2017 
a precios de mercado de acuerdo con INE: Cataluña, Madrid, Andalucía, Comunidad Valenciana y País Vasco.

En lo referente a los objetivos específicos que se conjugan con la idea de poder dar respuesta al objetivo general, la investigación ha desarrollado los siguientes:

- Definir los requerimientos y exigencias de las ofertas de trabajo publicadas en las principales webs de ofertas de empleo, en el sector del marketing B2C, con respecto a las habilidades técnicas exigidas en las mismas.

- Definir los requerimientos y exigencias de las ofertas de trabajo publicadas en las principales webs de ofertas de empleo, en el sector del marketing B2C, con respecto a las metahabilidades exigidas.

- Definir los requerimientos y exigencias de las ofertas de trabajo publicadas en las principales webs de ofertas de empleo, en el sector del marketing B2C, con respecto a los conocimientos fundamentales sobre el marketing.

- Definir la relación existente entre la experiencia requerida a los candidatos y la retribución ofrecida en las ofertas de trabajo publicadas en las principales webs de empleo, en el sector del marketing B2C.

\section{Hipótesis de la investigación}

Las hipótesis sobre las que se realizará esta investigación se han redactado de acuerdo a los aspectos más significativos del estado de la cuestión, que han sido expuestos en el marco teórico:

H1: Las habilidades propias de la digitalización serán el requerimiento más representativo de las ofertas de empleo para graduados en marketing en el mercado B2C.

Por la relación tan estrecha en el marketing entre habilidades técnicas y digitales, el desarrollo de esta hipótesis ayudará a generar respuestas al objetivo específico relacionado con las habilidades técnicas.

H2: Las competencias derivadas del conocimiento sobre los fundamentos del marketing tendrán un alto nivel de exigencia para los graduados en marketing en el mercado B2C.

Con esta hipótesis se podrá comprobar la veracidad del tercer objetivo específico de los que componen la investigación, dado que si se determina el nivel de exigencia sobre los conocimientos de los fundamentos de marketing, se inferirá de ello los requerimientos referidos en el objetivo mencionado.

H3: Existe una estrecha relación entre las exigencias de experiencia de la oferta para graduados en marketing, y la remuneración propia del puesto de trabajo ofertado en el mercado B2C. La probatura o no de esta hipótesis permitirá en cualquier caso establecer la relación existente entre remuneración y experiencia, tal y como queda contemplado en el objetivo específico tres. 
El segundo objetivo quedaría cubierto por las dos primeras hipótesis, por cuanto la relevancia y el posicionamiento de las categorías correspondientes a las habilidades técnicas y a los fundamentos del marketing no se entienden si no es en su interacción con la tercera y última: las metahabilidades.

Asimismo, a partir de estas hipótesis que dan respuesta a los objetivos específicos, se logrará también dar respuesta al objetivo general de la investigación.

\section{Metodología}

La presente investigación surge de la observación y el análisis de las competencias, habilidades y conocimientos requeridos en las ofertas de empleo en marketing anunciadas en los principales portales en España dedicados a la contratación. Estas ofertas han sido extraídas mediante el uso de la técnica Desk Research para pasar posteriormente a la realización de un análisis de contenido mediante la categorización y el etiquetaje de los datos observados. Una vez que estos han sido ordenados, categorizados y etiquetados, el trabajo se ha centrado en la cuantificación de las observaciones con el fin de observar la importancia que el mercado de empleo en el sector B2C concede a cada una de las categorías.

Se trata por tanto de una investigación de carácter descriptivo y de naturaleza mixta al usar tanto técnicas cualitativas, en lo que concierne a la búsqueda y elevación de la información, como cuantitativas en lo referente a la contabilización de los datos.

El procedimiento desarrollado para llegar a la extracción de los datos sobre los que se fundamentará el posterior análisis se ha generado en diferentes etapas.

En primer lugar se ha procedido a la selección de las comunidades autónomas de España en las que se quiere desarrollar la investigación. Esta selección se ha realizado atendiendo a aquellas cinco comunidades autónomas con mayor actividad económica, tal y como se observa en el gráfico 1.

El proceso de búsqueda se ha realizado online debido al gran impulso que ha adquirido esta modalidad de búsqueda de empleo que permite buscar trabajo mediante la técnica Desk Research, facilitando mucho la labor.

Posteriormente se pasa a determinar aquellos portales web que adquieren más notoriedad en la publicación de ofertas de empleo. Para ello se han sometido a observación los portales más destacados: Infojobs, Infoempleo, Laboris y Monster. Este proceso de observación se llevó a cabo entre los meses de diciembre de 2017 y abril de 2018, llegando a la conclusión de que los portales Infojob e Infoempleo son los de mayor número de ofertas publicadas, siendo por lo tanto aquellos en los que las empresas depositan mayoritariamente su confianza. 
Gráfico 1. Número de ofertas recogidas por comunidades autónomas vs. PIB 2017 (precios de mercado)

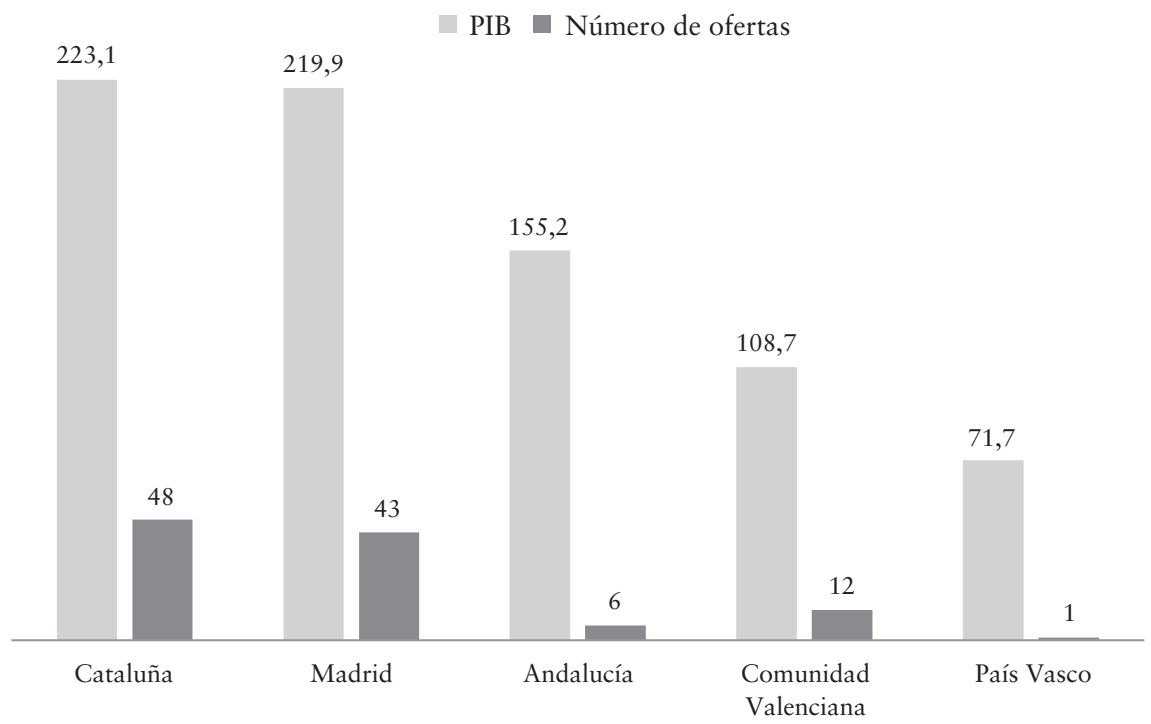

Fuente: Elaboración propia a partir de datos del INE.

Tabla 1. Portales empleados y número de ofertas

\begin{tabular}{cc}
\hline Portales usados para búsquedas & Total de ofertas en 4 meses de búsqueda \\
\hline Infojobs & 98 \\
\hline Infoempleo & 12 \\
\hline Laboris & 0 \\
\hline Monster & 6 \\
\hline
\end{tabular}

Fuente: Elaboración propia.

En efecto, como puede observarse en la tabla 1, las diferencias en lo que tiene que ver con el número de ofertas publicadas en esos cuatro meses es muy elevada, quedando el portal Infojobs muy distanciado de todos los demás con 98 resultados, seguido por Infoempleo con 12 . Viendo estos datos, podría decirse que la notoriedad de portales como Laboris o como Monster en el campo de las ofertas de empleo en marketing es testimonial.

La selección de las diferentes piezas que componen la investigación se ha desarrollado de acuerdo a una serie de filtros que determinan, una vez que estos se cumplen, la idoneidad de la oferta de empleo para ser introducida dentro de la investigación: 
1) Requerimiento del grado o licenciatura de marketing.

2) Grado o licenciatura en marketing no requerido pero valorado.

3) El campo de actividad de la empresa debe ser el B2C, quedando esto de manifiesto en el propio anuncio o bien a través de la información ofrecida en la web corporativa de la compañía en cuestión.

4) Las ofertas seleccionadas tienen que ser para las siguientes comunidades autónomas: Cataluña, Madrid, Andalucía, Comunidad Valenciana y País Vasco.

Para la búsqueda de las ofertas en los diferentes portales web de empleo se ha utilizado 'marketing' como palabra clave. Los resultados obtenidos han sido seleccionados como piezas para el análisis de contenido en función de su adaptación a los requisitos arriba mencionados.

Una vez seleccionados aquellos portales web sobre los que se desarrollará el mencionado análisis de contenido, se procede a la extracción de resultados de los mismos. Para ello se ha utilizado la plantilla diseñada por Pefanis y Harich (2010) en un estudio de características similares desarrollado en Estados Unidos. Esta tabla, que se toma como modelo organizativo de información, será adaptada en función de los objetivos y necesidades de este estudio. Al utilizar este tipo de tablas para el análisis, la investigación consigue reunir los aspectos de objetividad y sistematización, y logra, de acuerdo con Krippendorff (1990; en Andreu, 2000), generar reglas explícitas de investigación y aplicables a todas las unidades de análisis: objetividad, sistematicidad y cuantificación (Berelson, 1952). El resultado será de inferencias reproducibles que pueden ser aplicadas en su contexto (Krippendorff, 1990). Se trata por tanto del análisis de los mensajes manifiestos y latentes de un cuerpo de contenido (Krippendorff, 2013; McQuail, 2013). En definitiva, una gran cantidad de datos que puedan ser ordenados minuciosamente por categorías (Igartua, 2006).

Sí es importante señalar que la investigación ha requerido una constante modificación de la tabla de Pefanis y Harich (2010), pues en la medida en que se ha ido desarrollando el trabajo de extracción de piezas, y su posicionamiento tras el análisis en las diferentes categorías establecidas, la investigación ha exigido incrementar o completar las originales a fin de adaptar la información. La adaptación de la que se habla es necesaria, pues los ocho años transcurridos desde la publicación de dicha plantilla han generado cambios en las competencias, las habilidades del marketing y por tanto en los requisitos de contratación. Sí se han respetado las categorías iniciales en las siguientes dimensiones: Portal, Empresa, Puesto requerido, Origen geográfico y Experiencia como datos fundamentales de clasificación y las categorías generales: Habilidades técnicas; Metahabilidades; Conocimiento sobre fundamentos de marketing. A éstas se ha añadido la categoría de la remuneración que ayuda a darle valor económico al puesto y a la actividad requerida y que incluye las siguientes horquillas: remuneración entre 10.000 y 20.000, entre 21.000 y 30.000, entre 31.000 y 45.000, Más de 45.000 y No especificada.

La adaptación de la tabla de Pefanis y Harich se ha hecho a partir de los resultados del propio análisis de contenido, de tal manera que se han añadido los nuevos 
ítems necesarios para el análisis según han ido apareciendo en el texto de la pieza. Así, se han introducido etiquetas propias de esta investigación como la comunidad autónoma en que se realiza la oferta, el número de años de experiencia requeridos al candidato/a o la remuneración. En lo que tiene que ver con las habilidades técnicas, a las de Pefanis y Harich se han añadido las siguientes: CMS, HTML, Diseño gráfico, Inbound MKT, e-mailing, MKT de afiliación, Growth Hacking, Google Adwords, Redes Sociales, Photoshop, Java, Word Press, Adobe, Mobile MKT. En Metahabilidades se incorporan: flexibilidad, inglés, otros idiomas, organización y planificación, trabajo por equipos, capacidad analítica, proactividad, gestión de proyectos, gestión de influenciadores, eventos. En la sección correspondiente a conocimientos sobre fundamentos de MKT se añaden: estrategia de formación, estrategia de comunicación, gestión de clientes, MKT industrial, revenue MKT, trade MKT, knowledge in retail. A toda esta información se ha sumado aquella que tiene que ver con la experiencia-remuneración, en función de los aspectos referentes a este tema observados en el análisis.

\section{Resultados}

El análisis de los datos se ha dividido para su estudio en cuatro categorías, a saber: requerimientos relativos a las habilidades técnicas, a las metahabilidades, a los conocimientos sobre los fundamentos del marketing y al binomio experiencia-remuneración.

\subsection{Habilidades técnicas}

Tal y como se observa en el gráfico 2, son 22 las competencias encontradas en la oferta de empleo respecto de esta primera categoría denominada habilidades técnicas. Un abanico que va desde las últimas habilidades producto de la digitalización, tales como Growth Hancking o Inbound marketing, hasta aquellas otras más clásicas y que coexisten con las primeras: MS Office o CRM.

Las cinco competencias más demandadas a los graduados en marketing son, por este orden, las siguientes: gestión de las Redes sociales, el SEO y SEM, el paquete de MS Office, Google Analytics y e-mailing, algo que concuerda con investigaciones anteriores como la de Leeflang et al. (2014) al señalar a las herramientas y tecnologías digitales como una de las competencias más requeridas a los profesionales del marketing. En otras palabras, lo que más interesa a la empresa en esta categoría, además de garantizarse el uso correcto de las herramientas básicas en el puesto ( $M S$ Office) es el posicionamiento en motores de búsqueda (SEO y SEM), la comunicación con el cliente (e-mailing) y la medición de los resultados sobre las acciones que la compañía desarrolle (Google Analytics).

De otra parte, competencias tales como el software para el análisis de datos, el Growth Hacking, el Word Press, el paquete de Adobe y el Mobile marketing se 
presentan como las menos requeridas, si bien se debe considerar su importancia como habilidades emergentes, principalmente el Growth Hacking, Word Press y Mobile marketing.

Finalmente, cabe señalar dos cuestiones. Por un lado, el exiguo porcentaje del 0,8\% alcanzado por el Mobile marketing, competencia que sin embargo, a juicio de los investigadores, tendrá una relevante demanda por las empresas muy pronto. De otro lado, y aunque con valores muy discretos salvo en el caso del MS Office (12\%), es significativa la convivencia de competencias técnicas altamente demandadas en el marketing del siglo Xxi, como el CRM, la Minería de datos, el Photoshop o el MS Office, con las habilidades propias de la implantación de las últimas tecnologías.

Gráfico 2. Habilidades técnicas requeridas por la oferta en el mercado B2C [frecuencia relativa $(\%)$ ]
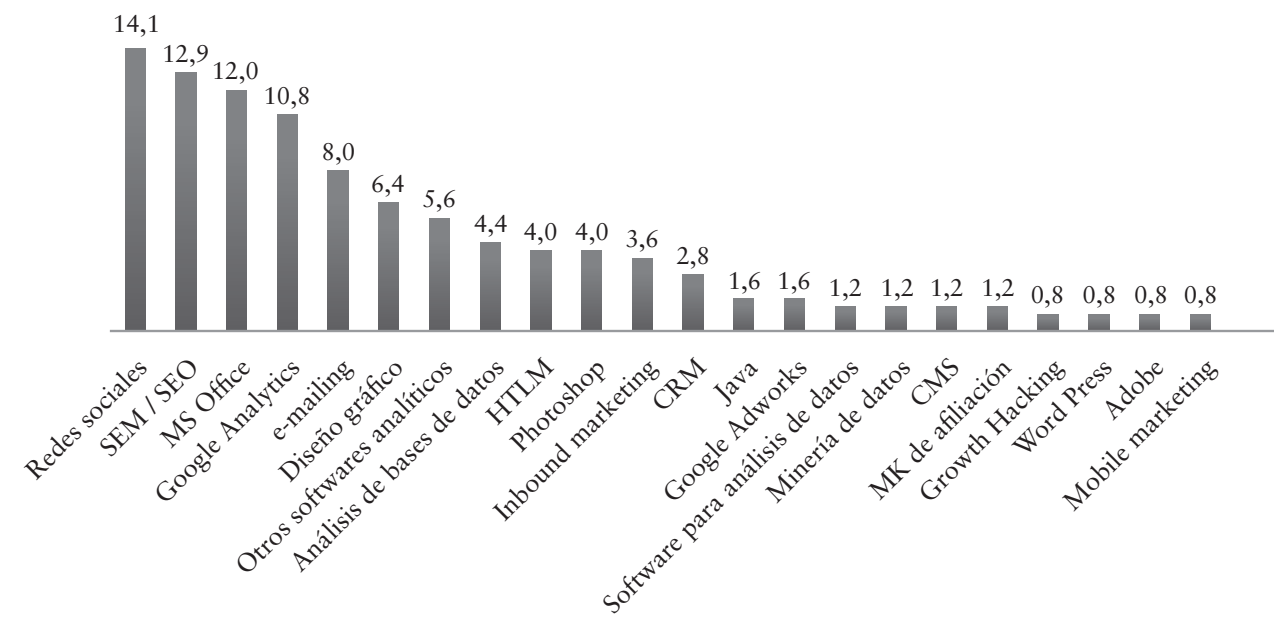

Fuente: Elaboración propia.

\subsection{Metahabilidades}

A la luz de los datos aportados por el gráfico 3, se han detectado un total de 19 competencias para la categoría de metahabilidades, entre las que destaca el grupo formado por el idioma inglés, la comunicación escrita, la oral, otros idiomas y el trabajo en equipo, por este orden. Se observa por tanto como máxima preocupación todo lo concerniente a las habilidades comunicativas en general, algo que coincide con investigaciones anteriores (Pefanis y Harich, 2010; Wroblowská, 2016).

De otra parte, las competencias a las que menos relevancia se ha concedido han sido el comportamiento ético, el comportamiento proactivo, la flexibilidad y el liderazgo. 
Gráfico 3. Metahabilidades requeridas por la oferta en el mercado B2C [frecuencia relativa $(\%)$ ]

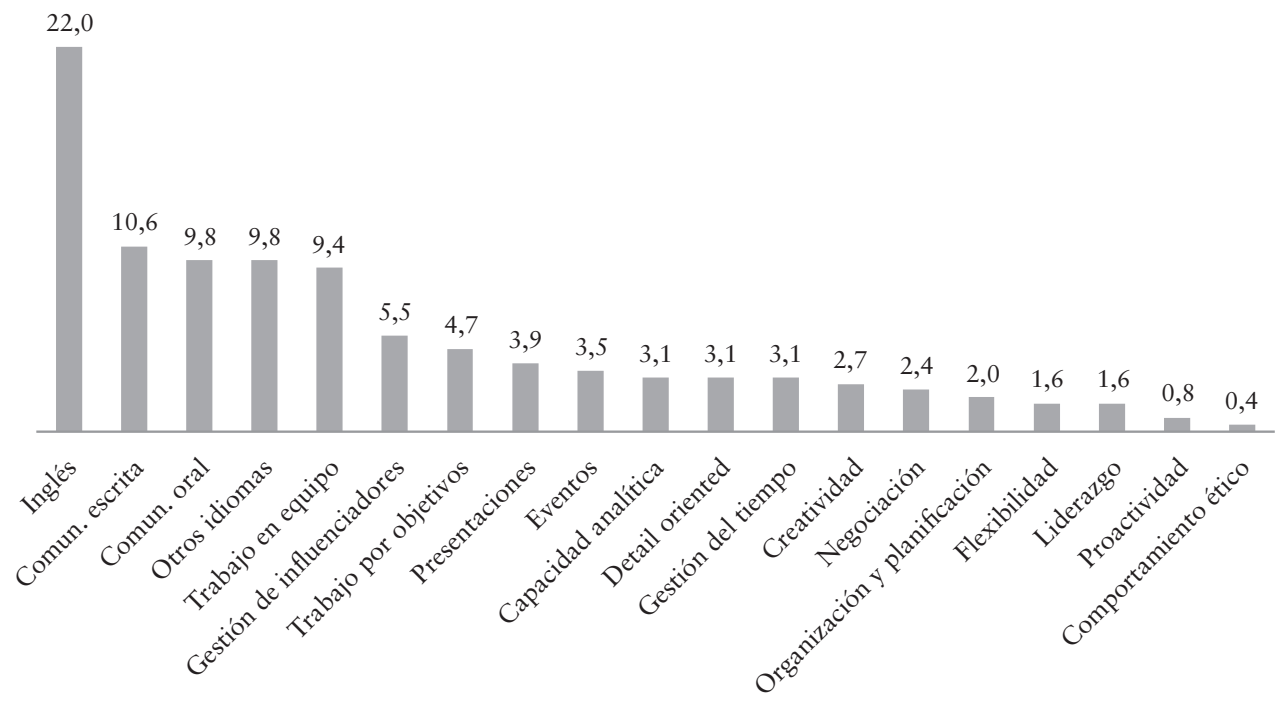

Fuente: Elaboración propia.

Asimismo, cabe destacar la aparición de una nueva metahabilidad como es la gestión de influenciadores, la cual no se incluye en ninguna de las investigaciones realizadas hasta la fecha. Dicha competencia cobra sentido por el uso creciente de las redes sociales y blogs, así como por su importancia para el posicionamiento de las marcas a través de la comunicación con su público objetivo.

\subsection{Conocimientos sobre fundamentos del marketing}

Tal y como se desprende de los datos aportados por el gráfico 4 , se han recogido un total de 26 competencias demandadas por la empresa en esta categoría, denominada conocimientos sobre fundamentos del marketing.

Las competencias más solicitadas han sido, por este orden, las siguientes: plan de marketing, marketing digital, campañas publicitarias, conocimientos del sector específico y gestión comercial. De estos datos se desprenden dos cuestiones de interés. De una parte, la exigencia al candidato del dominio del microentorno, representado tanto por las campañas publicitarias dirigidas al consumidor, como por los conocimientos sobre el sector o por la gestión comercial, entendida como labor sobre el cliente. De otra parte, se observa cómo las habilidades técnicas penetran también en esta tercera categoría por la existencia del componente digital requerido en los planes de marketing y en las campañas publicitarias. 
Gráfico 4. Habilidades requeridas por la oferta referidas a los conocimientos sobre fundamentos del marketing en el mercado B2C [frecuencia relativa (\%)]

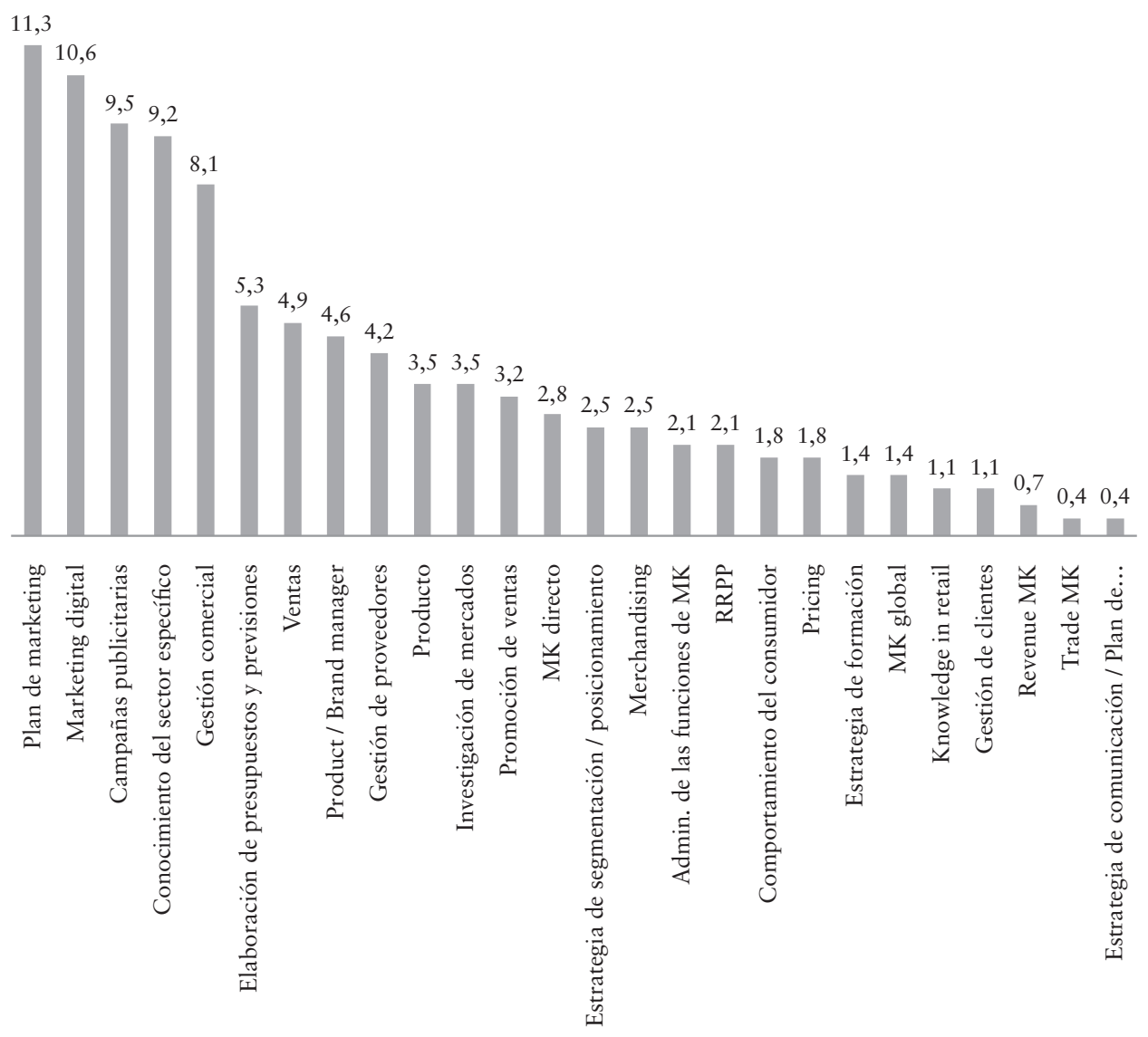

Fuente: Elaboración propia.

En sentido contrario, las competencias menos demandadas se han referido a la estrategia de comunicación, al trade marketing, al revenue marketing, a la gestión con clientes y al knowledge in retail.

Se desprende de estos datos el bajo interés despertado por aquellas habilidades en torno a la función de distribución, sobre la que apenas aparece algún reflejo representado por el knowledge in retail, trade marketing o la promoción de ventas -esta última mejor valorada-, centrada en estrategias de push.

La función del precio se encuentra representada con un $1,8 \%$ en una posición bastante discreta, mientras que el interés por el producto, caracterizado por las competencias de producto y product y brand manager crecen hasta el 3,5\% y 4,6\%, respectivamente. Por su parte, la función de comunicación se encuentra fuertemente representada en competencias tales como las propias campañas publicitarias, la promoción 
de ventas, el marketing directo o las RRPP; situación esta corroborada por estudios anteriores como los de Pefanis y Harich (2010) o Carson y Gilmore (2000).

Asimismo, la comercialización también se encuentra bien representada en competencias requeridas tales como la gestión comercial o las ventas; habilidades no tenidas en cuenta en la literatura consultada como requerimientos de la oferta de empleo para graduados en marketing.

\subsection{Correlación experiencia-remuneración}

A la luz de los datos aportados por la investigación y trasladados a la tabla 2 y gráfico 5 , se pueden observar varios aspectos significativos. Así, la experiencia más solicitada por las empresas es la de 2 y 3 años, siendo la de 4, 5 o más la menos demandada.

Asimismo, solo se han encontrado 10 ofertas en las que no se requiere experiencia, suponiendo tan solo el $9 \%$ del total de anuncios. Si a este dato sumamos las ofertas en las que se requiere un año de experiencia, la cuantía alcanza el $26 \%$ de la oferta total. Esta parte de la oferta laboral representaría aquella a la que tendrían acceso los recién graduados en marketing que buscan alcanzar su primer empleo una vez realizadas las prácticas.

Tabla 2. Correlación experiencia-remuneración

\begin{tabular}{lcccccccc}
\hline $\begin{array}{l}\text { Remuneración } \\
\text { ofrecida }(€)\end{array}$ & $\begin{array}{c}\text { No } \\
\text { requerida }\end{array}$ & 1 año & 2 años & 3 años & 4 años & 5 años & $\begin{array}{c}\text { más de } \\
5 \text { años }\end{array}$ \\
\hline $10.000 / 20.000$ & 5 & 3 & 4 & 5 & 1 & 0 & 1 & 19 \\
\hline $21.000 / 30.000$ & 0 & 4 & 7 & 0 & 1 & 4 & 2 & 18 \\
\hline $31.000 / 45.000$ & 0 & 0 & 0 & 1 & 1 & 2 & 0 & 4 \\
\hline Más de 45.000 & 0 & 0 & 0 & 0 & 0 & 0 & 1 & 1 \\
\hline No especificada & 5 & 12 & 21 & 22 & 3 & 4 & 1 & 68 \\
\hline & 10 & 19 & 32 & 28 & 6 & 10 & 5 & 110 \\
\hline
\end{tabular}

Fuente: Elaboración propia.

También se comprueba (véase tabla 2 o gráfico 5) cómo las remuneraciones más altas se corresponden con aquellos perfiles de mayor experiencia. Así, los honorarios por encima de los $45.000 €$ están solo al alcance de perfiles cuya experiencia es superior a los 5 años, si bien es cierto que también encontramos en este rango tres ofertas con una remuneración media-baja y baja. 
En conclusión, los perfiles con experiencia de más de 5 años cuentan con una horquilla salarial más amplia alcanzando remuneraciones de más de $45.000 €$, mientras que los perfiles de 1 y 2 años de experiencia tienen una horquilla más estrecha, la cual no supera los $30.000 €$.

Respecto a la remuneración, tomada esta de forma aislada, se comprueba que el nivel salarial más ofrecido es el que queda comprendido en la horquilla entre 10.000 y $20.000 €$, el rango más bajo, algo que contrasta con los valores aportados por la consultora Randstad (2018), quien ofrece unos datos ampliamente superiores a los registrados en el presente estudio.

Gráfico 5. Correlación experiencia-remuneración: número de ofertas (frecuencia relativa)

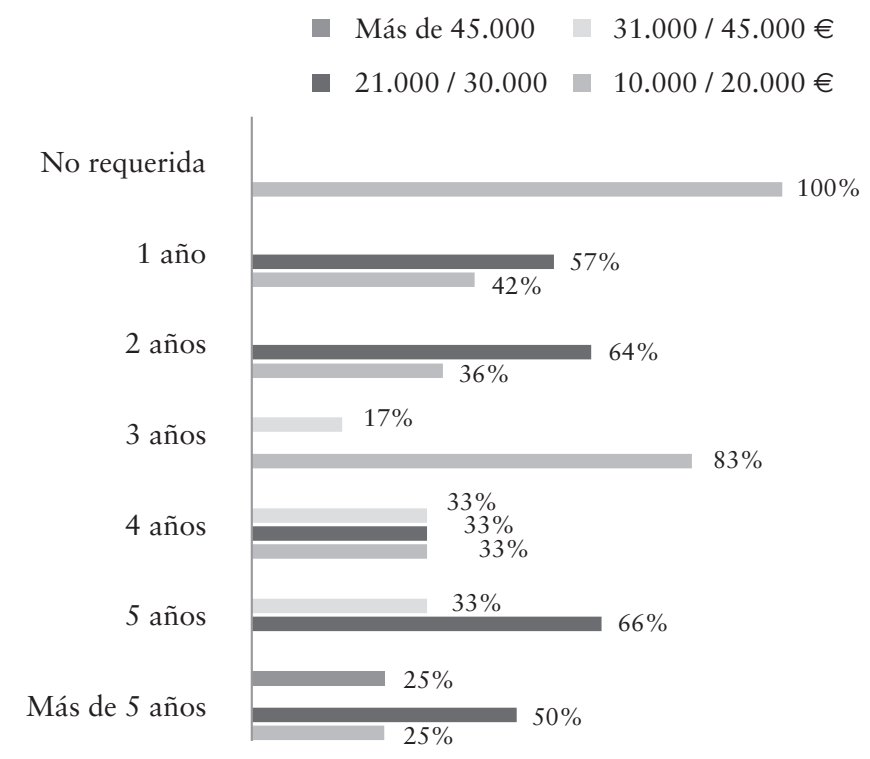

Fuente: Elaboración propia.

Para finalizar, la oferta de empleo más repetida es aquella que combina los 2 años de experiencia con una remuneración de 21.000 a $30.000 €$.

\section{Discusión e implicaciones}

Dado el objetivo general de la investigación, dirigido a la definición de las competencias exigidas a los graduados en marketing en el mercado B2C, explicitadas mediante las ofertas laborales publicadas en los principales portales de empleo, y dados los resultados de la investigación expuestos anteriormente, se han alcanzado las siguientes conclusiones: 
Las competencias más buscadas en los profesionales son las que aparecen en la tabla 3 .

Tabla 3. Top ten de las competencias más requeridas en el mercado, aparecidas en las ofertas de empleo

\begin{tabular}{lc}
\hline \multicolumn{1}{c}{ Competencias } & N. $^{\circ}$ de veces solicitada \\
\hline Idioma inglés & 56 \\
\hline Redes sociales & 35 \\
\hline Plan de marketing & 32 \\
\hline SEO/SEM & 32 \\
\hline Marketing digital & 30 \\
\hline MS Office & 30 \\
\hline Campañas publicitarias & 27 \\
\hline Comunicación escrita & 27 \\
\hline Google Analytics & 27 \\
\hline Conocimiento del sector específico & 26 \\
\hline
\end{tabular}

Fuente: Elaboración propia.

En lo que a la categoría sobre habilidades técnicas respecta, se ha comprobado cómo la incorporación de nuevas competencias producto del desarrollo digital coexiste con otras habilidades aparecidas en el pasado siglo Xx, algo que concuerda con las conclusiones de anteriores investigaciones (García y Echegaray, 2018).

Asimismo, el perfil buscado dentro de la mencionada categoría es el de un profesional con amplia formación en el marketing digital, especialmente en los campos correspondientes a las redes sociales, posicionamiento en motores de búsqueda y uso de las herramientas de Microsoft Office.

Además de lo dicho se comprueba cómo de las 22 competencias detectadas en la oferta de empleo en relación a esta categoría denominada habilidades técnicas, 12 son producto de la digitalización, es decir, habilidades que han irrumpido recientemente y que hace tan solo unos años no aparecían en prácticamente ninguna oferta de empleo (RRSS, SEO/SEM, Google Analytics, otros softwares analiticos, CMS, Inbound marketing, Growth Hacking, marketing de afiliación, e-mailing, Java, Word Press o Mobile marketing). A este respecto, se ha de señalar también cómo otras competencias digitales emergen en las otras dos categorías. Así, la gestión de influenciadores dentro de la categoría de metahabilidades o el marketing digital y las campañas de publicidad, en su parte digital, dentro de los conocimientos sobre 
fundamentos del marketing, son claramente nuevas competencias producto de la digitalización en la que vive la empresa.

Por todo ello se puede concluir en que las habilidades propias de la digitalización emergen como un requerimiento con mayor representación cada vez en las ofertas de empleo. Tanto el conjunto de competencias como su peso en relación al resto de habilidades lo ponen de manifiesto. Sin embargo, no es posible aún, a juicio de los investigadores, afirmar que las competencias propias de la digitalización se han conformado ya como el requerimiento más representativo de las ofertas de empleo para graduados en marketing del mercado B2C, aunque todo apunte a que lo harán en un futuro cercano. Hoy por hoy existen otros conjuntos o categorías de competencias todavía más requeridas que las digitales, como se verá después. Por tanto, no es posible de momento validar la hipótesis inicial descrita antes, si bien todo hace pensar que en tan solo unos pocos años se cumpla plenamente.

En relación a la segunda categoría analizada, metahabilidades, el perfil más buscado por el mercado coincide con el de un buen comunicador, con importantes competencias en idiomas - preferentemente inglés-, en comunicación escrita y oral, y bien capacitado para trabajar en equipo.

Respecto a la tercera categoría estudiada, el conocimiento sobre los fundamentos del marketing, cabe destacar en primer lugar la amplitud de la misma, dado que con 26 competencias es la más extensa. Además, dichas habilidades han sido requeridas un total de 283 veces en el conjunto de ofertas de empleo analizadas (véase gráfico 6), haciendo de esta categoría la de mayor peso, por encima de las metahabilidades y de las habilidades técnicas, respectivamente. Por consiguiente quedaría confirmada la hipótesis de partida según la cual las competencias derivadas del conocimiento sobre los fundamentos del marketing tienen un alto grado de requerimiento por las empresas.

Los requerimientos de las ofertas se dirigen fundamentalmente al dominio de los elementos que conforman el microentorno, representados por competencias como el conocimiento del sector específico, la gestión comercial o las campañas publicitarias.

Por otro lado, se ha constatado un bajo interés por las habilidades derivadas de la distribución y del precio, en contraposición a lo sucedido con las de la comunicación y el producto.

El análisis realizado sobre la correlación entre experiencia y remuneración ha dejado claros varios aspectos. Así, se ha comprobado cómo la experiencia laboral de 2 o de 3 años es la más solicitada por las empresas, alcanzado el $54 \%$ del conjunto de ofertas de empleo seleccionadas.

Asimismo, se concluye cómo aquellos graduados que intentan entrar por primera vez al mercado laboral, nos referimos a los alumnos que han concluido sus estudios recientemente, o a aquellos otros que han terminado con éxito sus prácticas profesionales, ven reducidas sus opciones de una manera significativa, pues las empresas dispuestas a contratar trabajadores con tan solo 1 año de experiencia, o incluso sin ella, representan tan solo una cuarta parte de la oferta ( $26 \%$ exactamente), obviamente con las remuneraciones más bajas. 
Gráfico 6. Número de veces que ha sido solicitada cada una de las tres categorías en la muestra de ofertas de empleo seleccionadas, como sumatorio del conjunto de solicitudes de cada una de las competencias que incluye

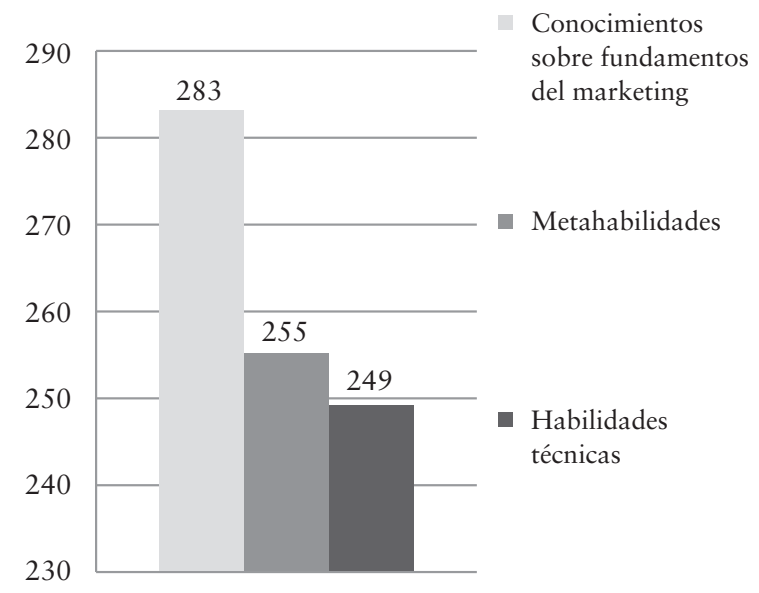

Fuente: Elaboración propia.

Para finalizar, se ha comprobado también cómo aquellas ofertas que requieren una experiencia mayor: 4, 5 o más años, son las mejor remuneradas, si bien cabe subrayar la paradoja que se da en el caso de la experiencia superior a 5 años, donde encontramos las mayores remuneraciones ofrecidas en todo el rango de experiencias, a la vez que un amplio porcentaje de ofertas con una remuneración media-baja y baja.

Por todo ello podemos afirmar que existe una estrecha relación entre los requerimientos de experiencia y la remuneración ofrecida, de forma que cuando aumenta la primera, crece también esta última, tal y como se estableció en las hipótesis contempladas anteriormente por esta investigación.

El presente estudio no está exento de algunas limitaciones que citamos a continuación. Dado que este trabajo se ha centrado en el mercado B2C, las conclusiones obtenidas no son extensibles de forma directa a otros mercados como pueda ser el B2B o el B2A. En consecuencia, dichos mercados podrían constituir un objeto de investigación relevante con arreglo a determinar la importancia y el alcance que su idiosincrasia pudiese tener sobre las habilidades y competencias demandadas a los graduados en marketing.

Finalmente, los resultados aportados por esta investigación pueden ser útiles a los centros de formación en marketing con objeto de reconducir sus planes de estudio, creando nuevas menciones o especializaciones en sus grados, teniendo en cuenta para ello los requerimientos formulados por las empresas en el mercado B2C. 


\section{Bibliografía}

\section{Artículos}

Andreu, J. (2000). "Las técnicas de análisis de contenido: una revisión actualizada”. Fundación Centro de Estudios Andaluces, Universidad de Granada, 10, (2), 1-34.

Brady, M., Fellenz, M. R., y Brookes, R. (2008). "Researching the role of information and communications technology (ICT) in contemporary marketing practices”. Journal of Business and Industrial Marketing, 23 (2), 108-114. http:// dx.doi.org/10.1108/08858620810850227

Bronwyn, F. (2003). "Un mundo de salarios". Harvard Business Review, 81, (11), 35-46.

Carbonell-Foulquie, P., Manuera-Aleman, J. L., y Rodríguez-Escudero, A. I. (2004). "Criteria employed for go/no-go decisions when developing successful highly innovative products”. Industrial Marketing Management, 33, (4), 307-316. http://dx.doi.org/10.1016/S0019-8501(03)00080-4

Carson, D. (1990). "Some exploratory models for assessing small firms marketing performance". European Journal of Marketing, 24, (11), 8-51.

Carson, D., y Gilmore, A. (2000). “SME Marketing Management competencies”. International Business Review, 9, (3), 363-382. http://dx.doi.org/10.1016/ S0969-5931(00)00006-8

Chiesa, C., y Renart, L. G. (2000). "La remuneración de redes comerciales en España”, Harvard-Deusto Marketing \& Ventas, 38, 64-70.

Cicera i Soler, J. (2009). "Remuneración comercial ajustada a la estrategia”. Harvard-Deusto Marketing \& Ventas, 91, 74-79.

Davis, R., Misra, S., y Van Auken, S. (2002). “A gap analysis approach to marketing curriculum assessment: A study of skills and knowledge”. Journal of Marketing Education, 24, 218-224 http://dx.doi.org/10.1177/0273475302238044

Day, G. S. (2011). “Closing the marketing capabilities gap”. Journal of Marketing, 74, (5), 183-195. https://doi.org/10.1509/jmkg.75.4.183

García, J., y Echegaray, L. (2018). "What competencies and skills should marketing professionals posses? An exploratory approach from a professional and academic perspective". Esic Market Economics and Business Journal, 49, (2), 239-260.

Gilmore, A., Carson, D., y Grant, K. (2001). "SME marketing in practice". Marketing, intelligence \& Planning, 19, (1), 6-11. http://dx.doi.org/10.1108/ 02634500110363583

Harraway, J. A., y Barker, R. J. (2005). "Statistics in the workplace: A survey of use by recent graduates with higher degrees". Statistics Education Research Journal, 4, 43-58.

Leeflang, P., Dahlström, P., Verhoef, P., y Freundt, T. (2014). "Challenges and solutions for marketing in a digital era”. European Management Journal, 32, (1), 1-12. http://dx.doi.org/10.1016/j.emj.2013.12.001 
McNally, R., Durmusoglu, S., y Calantone, R. (2013). "New Product Portfolio Management Decisions: Antecedents and Consequences”. Journal of Product Innovation Management, 30, (2), 245-261. http://dx.doi.org/10.1111/j.1540-5885.2012. 00997.x

Micu, A. C., Dedeker, K., Lewis, I., Moran, R., y Netzer, O. (2011). “The shape of marketing research in 2021". Journal of Advertising Research, 51, (1), 60-68. http://dx.doi.org/10.2501/JAR-51-1-213-221

Nomparte, V. (2003). “Tendencias en remuneración comercial”. MK. Marketing y Ventas para Directivos, 18, (182), 32-36.

Ortiz, M., Joyanes, L., y Giraldo, L. (2016). "Los desafíos del marketing en la era del big data”. E-Ciencias de la Información, 6, (1), 31. https://doi.org/10.15517/ eci.v6i1.19005

Pefanis, R., y Harich, K. (2010). "Knowledge and Skill Requirements for Marketing Jobs in the 21st Century". Journal of Marketing Education, 32, (3), 341-352. Doi: http://dx.doi.org/10.1177/0273475310380881

Royle, J., y Laing, A. (2014). “The digital marketing skills gap: developing a digital marketer model for the communication industries". International Journal of Information Management, 34 (2), 65-73. http://dx.doi.org/10.1016/j.ijinfomgt. 2013.11.008

Schibrowsky, J. A., Peltier, J. W., y Boyt, T. E. (2002). “A professional school approach to marketing education". Journal of Marketing Education, 24, 43-55. http://dx.doi.org/10.1177/0273475302241006

Torres, F., Tiffer, H., y Sandoval, Y. (2017). "Remuneración a la fuerza de ventas: variables que determinan su éxito o fracaso". TEC Empresarial. 10, (3), 41-49.

Tzokas, N., Hultink, E., y Hart, S. (2004). "Navigating the new product development process". Industrial Marketing Management, 33, pp. 619-626. http:// dx.doi.org/10.1016/j.indmarman.2003.09.004

Wroblowská, Z. (2016). "Requierements for Brand Managers and Product Managers Responsible for Competitiveness of Product and Brands". Journal of Conpetitiveness, 8, (3), 5-21.

Wroblowská, Z., y Ruda, T. (2015). “The Identification and Comparison of the Requirements Placed on Product Managers during the Recruitment Process". Journal of Competitiveness, 7, (3), 110-125. http://dx.doi.org/10.7441/joc.2015.03.08

\section{Libros e informes}

Bakhshi, H., y Mateos-García, J. (2012). Rise of the Datavores: How businesses analyse and use online data. London, NESTA. Recuperado de https://www.nesta. org.uk/publications/rise-datavores-how-uk-businesses-can-benefit-their-data

Berelson, B. (1952). Content Analysis in Communication Researches. Glencoe III, Free Press.

Boam, R., y Sparrow, P. (1992). "The rise and nationale of competency-based approaches”, en R. Boam y P. Sparrow, (eds.), Designing And Achieving Competency: a competency-based approach to managing people and organizations (pp. 3-11). Londres. McGraw-Hill. 
Dottle, C. (2018). "El mercado de selección en España. Panorama general”, en Guía del mercado laboral 2018. Recuperado de http://guiasalarial.hays.es/trabajador/ home

Foxize School (2014). El Director de Marketing del Futuro. Recuperado de https:// www.foxize.com/ebooks/el-director-de-marketing-del-futuro

Fuentes, J. (2009). Cooking sales: vender más y mejor. Madrid. Ibersaf Ediciones.

Igartua, J. J. (2006). Métodos cuantitativos de investigación en comunicación. Barcelona. Bosch.

INE (2018). España en cifras 2018. Recuperado de http://www.ine.es/prodyser/ espa cifras/2018/31/index.html\#zoom=Z

Krippendorff, K. (1990). Metodología del análisis de contenido. Teoría y práctica. Barcelona. Paidos.

- (2013). Content analysis: An introduction to its methodology. Thousand Oaks, CA. SAGE Publications.

McQuail, D. (2013). Journalisand Society. London. SAGE.

Page, M. (2018). Estudios de remuneración, recuperado de https://www.michaelpage. es/prensa-estudios/estudios/estudios-de-remuneracion

Palomo, M. T. (2008). El perfil competencial del puesto de directorla de marketing en organizaciones de la Comunidad de Madrid. Madrid. ESIC.

Randstad (2018). Informe de Tendencias salariales 2018, recuperado de https:// research.randstad.es/informe-de-tendencias-salariales-2018/

Santesmases, M. (2007). Marketing. Conceptos y estrategias. Madrid. Pirámide.

Ventura, J. (2013). Análisis estratégico de la empresa. Madrid. Ediciones Paraninfo. 


\section{Datos de los autores}

Nombre: Jorge García Martín

Cargo: Profesor Área de Marketing Estratégico

Escuela/Facultad:

Universidad: CÁMARABILBAO University Business School

Dirección: Licenciado Poza, 17, 48011 Bilbao

Teléfono: +34944702484

Correo electrónico: jorge.garcia@camarabilbaoubs.com

Nombre: Lázaro Echegaray

Cargo: Profesor Área de Sociología e Investigación de Mercados

Escuela/Facultad:

Universidad: CÁMARABILBAO University Business School

Dirección: Licenciado Poza, 17, 48011 Bilbao

Teléfono: +34 944702484

Correo electrónico: lazaro.echegaray@camarabilbaoubs.com 